\title{
Determination of valid $R$-curves for materials with large fracture toughness to yield strength ratios*
}

\author{
Y. W. MAI \\ Department of Mechanical Engineering, Imperial College of Science and Technology, London, UK
}

\section{A. G. ATKINS}

Delta Materials Research, Ipswich, Suffolk, UK

\section{R. M. CADDELL}

Department of Mechanical Engineering, University of Michigan, Ann Arbor, Michigan, USA

(Received March 21, 1975; in revised form January 30, 1976)

\begin{abstract}
Crack growth resistance curves are derived from a generalised theory of quasi-static crack propagation due to Gurney and Hunt. Both the subcritical and continuous cracking regions are investigated, where the fracture toughness of the material may depend on the cracking rate, the reacting environment at the crack tip and the mode of fracture. Precise conditions for stability of the spreading crack relative to chosen constraints of either a displacement- or load-controlled machine are formulated. Cracking of sheet materials with high fracture toughness and low yield stress, $\left(\right.$ e.g. $\left(K / \sigma_{y}\right)^{2}>200 \mathrm{~mm}$ ), which do not satisfy certain size requirements, is often complicated by generalised yielding at regions remote from the crack tip. Complete $R$-curves for such materials cannot be established with conventional testpieces in the laboratory. The present paper adopts a new experimental technique [1] where a laboratory size reinforcement rig attached to the testpiece eliminates all irreversibilities caused by generalised yielding. Valid fracture toughness values and crack growth resistance curves are thereby determined, irrespective of the amount of elastic and plastic deformations occurring at the crack tip. Successful $R$-curve experiments are described for fracture in a few ductile and tough materials such as 7075-T3. and 1100-0 aluminium alloys, and a low carbon steel. Comparison is made with other published $R$-curves, and the influence of sheet thickness and $\left(K_{\mathrm{Ic}} / \sigma_{y}\right)$ ratio on the geometry of $R$-curves is investigated. A simple relationship for $R$-curves is suggested, viz.: $R=R_{0}+F(\Delta L)^{p}$, where, it seems, $R_{0}$ can be identified with the plane strain toughness (i.e. $R_{0}=G_{\mathrm{lc}}=K_{\mathrm{lc}}^{2} / E\left(1-v^{2}\right)^{\frac{1}{2}}$ ). A possible reason for this unexpected result is given in the paper. Useful estimates of $K_{\mathrm{Ic}}$ may thus be available from thin sheet tests.
\end{abstract}

\section{Nomenclature}

\author{
$E$ : Young's modulus \\ $R$ : crack growth resistance \\ $\sigma_{y}: \quad$ yield stress \\ $L: \quad$ crack length \\ $K_{\mathrm{c}}$ : critical stress intensity factor \\ $H$ : half beam depth in DCB-type specimen \\ $A$ : crack area \\ $X$ : applied load \\ $u$ : displacement \\ A: strain energy function \\ $G$ : $\quad$ strain energy release rate \\ $\Omega$ : complementary strain energy function \\ $B$ : specimen thickness \\ $M$ : applied bending moment
}

* Paper presented in part at the 11th Annual Meeting of the Society of Engineering Science, November 11-13, 1974, Duke University, Durham, N.C., USA. 
$\theta$ : $\quad$ end slope

I: $\quad$ second moment of inertia

$W$ : total length of specimen

$x$ : horizontal co-ordinate used in beam on elastic foundation model

$v$ : vertical co-ordinate used in beam on elastic foundation model

$v_{\mathrm{c}}$ : critical relative opening displacement of beam at crack tip

$k$ : $\quad$ stiffness of half springs per unit length of beam

$\lambda$ : reciprocal of characteristic length of beam on elastic foundation

$r$ : length of unbroken ligament of testpiece

$h, d$ : cross-sectional area dimensions of test rig

I: Poisson's ratio

$K_{\mathrm{Ic}}:$ plane strain fracture toughness

$F, p$ : material constants defined in (18)

$\rho: \quad$ plastic zone size

\section{Introduction}

The fundamental concepts of $R$-curves and their use in predictions of critical cracking loads in sheet metals of various geometries have been described in ASTM STP No. 527. One interesting postulate is that crack growth resistance curves are a material property and independent of specimen geometry, for a given sheet thickness. In the 8th National Fracture Symposium at Brown University, Wang and McCabe [2] presented some experimental data showing consistent $R$-curves for CCT and CLWL specimens. However, such an idea is not yet conclusive $[3,4]$ and more experiments must be done to confirm this.

The establishment of a full $R$-curve (i.e. in both the sub-critical and continuous propagation regions) for a given material must involve the use of testpieces which promote stable cracking [5-8]. Many $R$-curve determinations have been performed on centre notched panels (which are inherently unstable $[5,7,8]$ ), so that it is not surprising that in such test only the initial part of the full $R$-curve has been obtained, i.e. only the "slow stable growth" region where the rising crack resistance to extension is opposed by the gradual development of the plastic zone at the crack tip. In the investigations of Heyer and McCabe [3] and Ripling and Falkenstein [9], CLWL and tapered DCB specimens were used. These have favourable geometric stability factors so that full $R$-curves were obtained in those studies. The point at which the $R$-curve gets cut off in testpieces that have unfavourable geometric stability factors varies with the particular geometry of the unstable testpiece, so that $K_{\mathrm{c}}$ values determined at such cut off points will vary from specimen type to specimen type. This is discussed more fully in Section 2 of this paper.

The slow growth period is represented by region 1 of the complete crack propagation $R$-locus shown in Fig. 1. It should be noted that in region 2, the subsequent continuous quasi-static crack growth at any instant may follow one of the three possible paths where $\mathrm{d} R / \mathrm{d} L \supseteqq 0$, depending on the applied stress fields and the material response to crack propagation in the fracture process. In fact, it has been demonstrated that during continuous crack spreading $R$ is rarely constant, as it may be affected by crack velocities, cycling of load or displacement, material thickness, specimen geometry, temperature and environment [6].

A further consideration (in addition to questions of stability) in the determination of $R$-curves for thin sheet materials-and indeed in the determination of fracture toughness in general-is that yielding at regions remote from the crack tip must be avoided. In other words, the net section stress in centre cracked panels or the bending stresses in the arms of DCB specimens, for example, must not be high enough to cause 


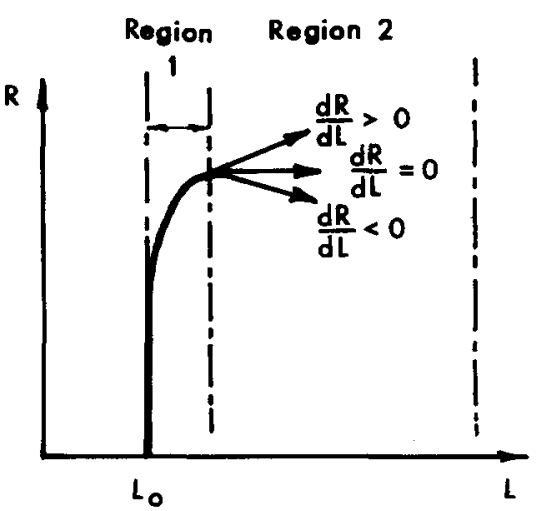

Figure 1. Typical crack propagation $R$-loci.

yielding. If such irreversibilities do occur, then it is in practice impossible to distinguish between the work of fracture (i.e. the source of $R$, concentrated at the crack faces) and the work of yielding. The adoption of LEFM formulae (with or without plastic zone corrections) to calculate $R$, in the presence of generalised yielding, is a dubious procedure because the system would not be "displacement reversible" after some crack propagation. This necessary condition of reversibility (upon which all LEFM is strictly predicated) has been emphasised in some publications [5-7, 11], but unfortunately has oftentimes been overlooked. When generalised yielding does occur, $R$-curves tend to be "too high", loads on the testpieces having to do yielding work as well as fracture work.

Thus, "full" $R$-curves that reflect only resistance to cracking must be designed to avoid generalised yielding. For a beam-like structure under longitudinal splitting of an existing crack, Gurney and Hunt [5], working from elementary beam theory, have shown that the necessary beam half depth $(H)$ for cracking before general yielding of the arms may be expressed as:

$$
H>3 E R / \sigma_{y}^{2} \text { or } H>3\left(K / \sigma_{y}\right)^{2} \text {. }
$$

Hahn, Sarrate and Rosenfield [10] have shown experimentally in DCB specimens that if yielding in parts other than at the crack tip is to be avoided, $H$ cannot be less that $1.5\left(K / \sigma_{y}\right)^{2}$, which is half the Gurney and Hunt estimation. Similarly there are minimum size requirements (in terms of $K / \sigma_{y}$ ) for ligaments in the paths of advancing cracks for different testpieces.

Inequalities such as (1) should not be confused with the minimum thickness requirement, $2.5\left(K_{\mathrm{Ic}} / \sigma_{y}\right)^{2}$ for "valid" plane strain tests. Such inequalities have to be satisfied for particular testpieces if LEFM ideas are to be applied to plane stress and "transitional" cracking experiments. It is apparent that the term $\left(K / \sigma_{y}\right)^{2}$ has as much significance in controlling transitions from cracking to general yielding of a structure, as in giving a physical measure of plastic zone sizes in plane stress or plane strain.

The $R$-curve technique has been used principally for cracking under conditions of plane stress in thin metal sheets for which $5 \mathrm{~mm}<\left(K / \sigma_{y}\right)^{2}<100 \mathrm{~mm}$ (e.g. see ASTM STP No. 527). Even so, it has not always been possible, in the particular specimens used, to eliminate generalised yielding. For more ductile and tough materials with $\left(K / \sigma_{y}\right)^{2}>200 \mathrm{~mm}$, general yielding other than merely at the crack tips is inevitable in conventional types of testpiece. The size requirement of inequality (Eqn. (1)) at large $\left(K / \sigma_{y}\right)^{2}$ is enormous.

Gurney, Mai and Owen [1] have recently developed a laboratory size apparatus for cracking materials with large $\left(K / \sigma_{y}\right)^{2}$ for a range of material thickness using small 
testpieces. Irreversibilities and large plasticity effects other than at the crack tips have been eliminated through reinforcements on the testpiece. In so far as the loading arms remain elastic this allows valid fracture toughnesses of the material to be generated without appreciable difficulties. The apparatus therefore simulates the large-scale quasibrittle behaviour of ductile materials which, in the usual laboratory testpieces, display the gross irreversibilities of "post yield" fracture. The apparatus is also extremely stable, in both hard, and even soft, testing machines, so that full $R$-curves are readily obtained.

In the present paper, complete $R$-curves for steel and aluminium samples, which include the slow stable growth and the continuous spreading regions, are discussed in terms of the $R-A$ diagrams. These results were obtained using the apparatus developed in [1]. Because of load capacity limitations imposed by the particular Instron testing machine that was available, experiments were restricted to the cracking of thin sheets, so that the data in [1] are obvious candidates for interpretation in terms of $R$-curves. However, the rig may be designed to accommodate very thick specimens. The results are compared with those of others, and we investigate the effect of thickness and $\left(K / \sigma_{y}\right)^{2}$ ratio on the geometry of the $R$-curves. An interesting possibility of deriving approximate $K_{\mathrm{lc}}$ values from $R$-curves is suggested.

\section{Theory of quasi-static cracking}

A general theory of quasi-static crack propagation has been proposed by Gurney [12] and subsequently extended in other papers with his co-workers [1, 5-7]. Plasticity effects are assumed to be limited to boundary layers of the cracked surfaces. "Quasi-static" is used when kinetic energies generated in the fracture process are small compared with the specific work of fracture. For an arbitrary crack embedded in an elastic solid body under the action of external forces $\left(X_{i}\right)$ with corresponding displacements $\left(u_{i}\right)$, the first law of thermodynamics gives:

$$
\Sigma X_{i} \mathrm{~d} u_{i}-\mathrm{d} A-R \mathrm{~d} A=0
$$

where $A$ is the nominal crack area and $A$ the strain energy function, single-valued in $(u A)$.

It can be easily obtained that:

$$
R=-(\partial \Lambda / \partial A)_{u_{i}}
$$

which is called the "specific work per unit area of cracked surfaces" or "the crack growth resistance". If we define the elastic strain energy release rate available for crack propagation as $G$, then we have:

$$
G=-(\partial \Lambda / \partial A)_{u_{1}}
$$

and coupled with (4), we get:

$$
G=R
$$

as the necessary condition for and during quasi-static crack extension. It can be shown that when $R<G$, unstable cracks will propagate with appreciable kinetic energies being generated.

Precise conditions governing the spreading of stable cracks relative to chosen constraints of either a load- or a displacement-controlled machine were first formulated by 
Gurney and Hunt [5] and, independently in different form, by Clausing [8]. For a recent pedagogical treatment see Gurney and Mai [7]. In a hard testing machine in which $\mathrm{d} u / u>0$, the criterion may be expressed as:

$$
\mathrm{d} R / \mathrm{d} A>(\partial G / \partial A)_{u} \quad \text { or } \quad \mathrm{d} R / \mathrm{d} A>-\left(\partial^{2} \Lambda / \partial A^{2}\right)_{\mu}
$$

and in a soft testing machine in which $\mathrm{d} X / X>0$ :

$$
\mathrm{d} R / \mathrm{d} A>(\partial G / \partial A)_{X} \quad \text { or } \quad \mathrm{d} R / \mathrm{d} A>\left(\partial^{2} \Omega / \partial A^{2}\right)_{X}
$$

where the complementary strain energy function $(\Omega)$ is related by $X u-\Lambda=\Omega$. The right hand quantities in expressions 6 and 7 are called the geometrical stability factors (gsf) and they determine the stability of cracks. It should be noted that $(\partial G / \partial A)_{u}$ or $(\partial G / \partial A)_{X}$ can be obtained readily for a given test geometry, by either classical elasticity solutions or experimental compliances. If the crack growth resistance $(R)$ as a function of $(u, A)$ or $(X, A)$ can be derived, the crack area $(A)$ at instability (and hence the critical values of $R$ or $K_{\mathrm{c}}$ ) will be determined from the inequality expressions in (6) and (7).

The geometry of the complete $R$-curve may be constructed from experiments performed

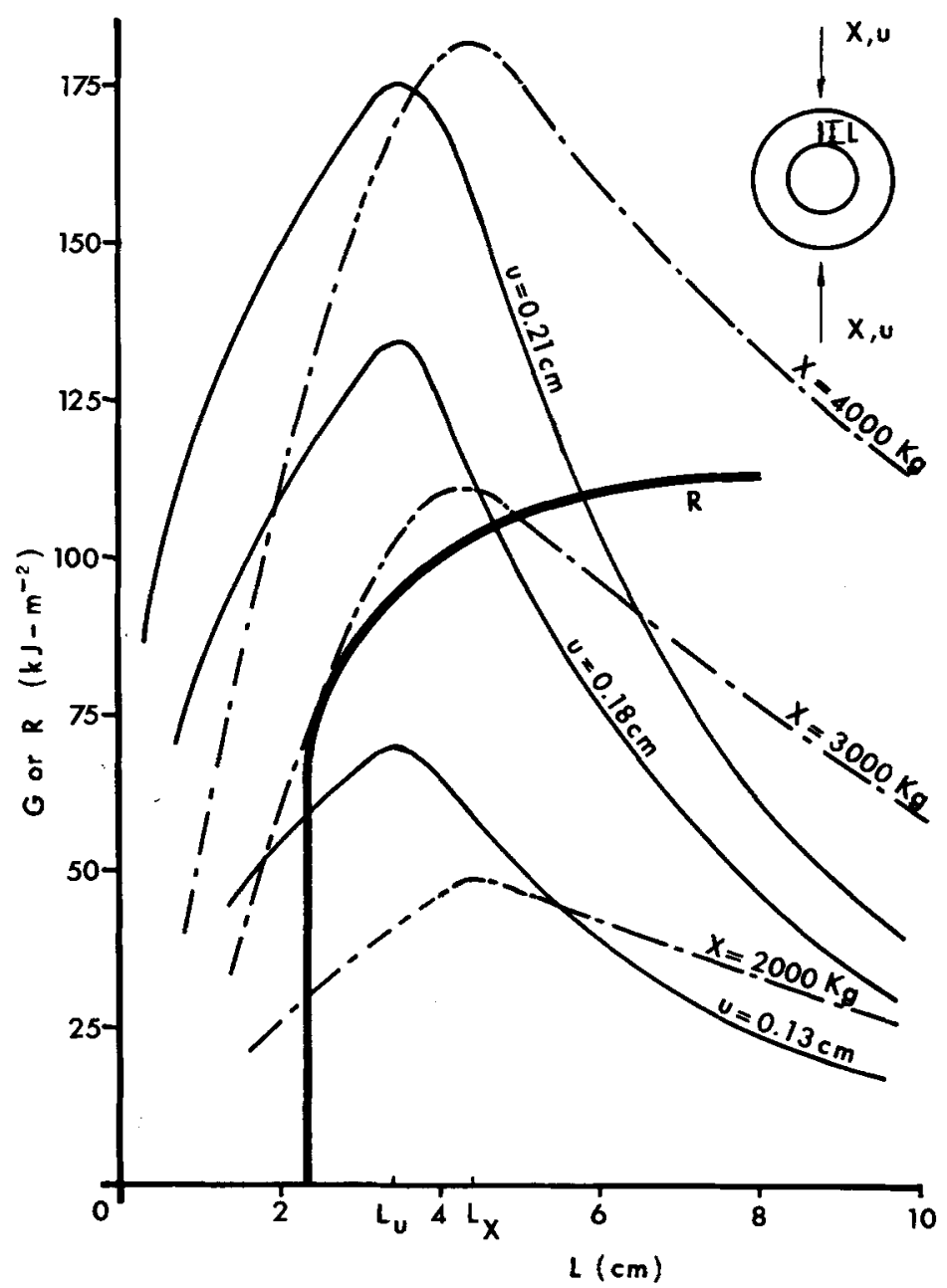

Figure 2. Cracking of a steel ring specimen under diametrical compression. 
on stable testpieces using (4) and (5). Theoretically, this is a 3-dimensional curve relating the quantities $(R, A, u)$ or $(R, A, X)$ in a form not yet defined. It has been postulated [13-16] that during slow stable subcritical growth in a sheet of a given thickness $B, R$ is a function of only $\Delta L$ and is independent of $L$. Crack spreading after the plastic zone has grown to an equilibrium maximum size is, however, dependent on mechanical variables such as test geometry, crack velocities, thickness and temperature.

The example of a cracked ring under diametrical compression [7] as shown in Fig. 2 demonstrates some of the more important concepts of $R$-curves and related problems on crack stability. By shifting the $R$-curve along the horizontal axis, it is clear that $R$ or $K_{\mathrm{c}}$ at instability is variant, and that to obtain a complete $R$-locus during crack extension, a minimum crack area $A_{X}=t L_{X}$ or $A_{u}=t L_{u}$ (corresponding to conditions of a soft and a hard testing machine, respectively) will be necessary. Since $(\partial G / \partial A)_{X}$ or $(\partial G / \partial A)_{u}$ is very much less than $\partial R / \partial A$ for $A>A_{X}$ or $A_{u}$, the complete crack propagation locus ( $R$-curve) for otherwise unstable materials can be easily obtained.

\section{A crack propagation apparatus for large $\left(K / \sigma_{y}\right)^{2}$ materials}

Figure $3 a$ shows the simple crack propagation apparatus developed in [1] and used in this work. The construction and detailed dimensions of the test rigs have been described

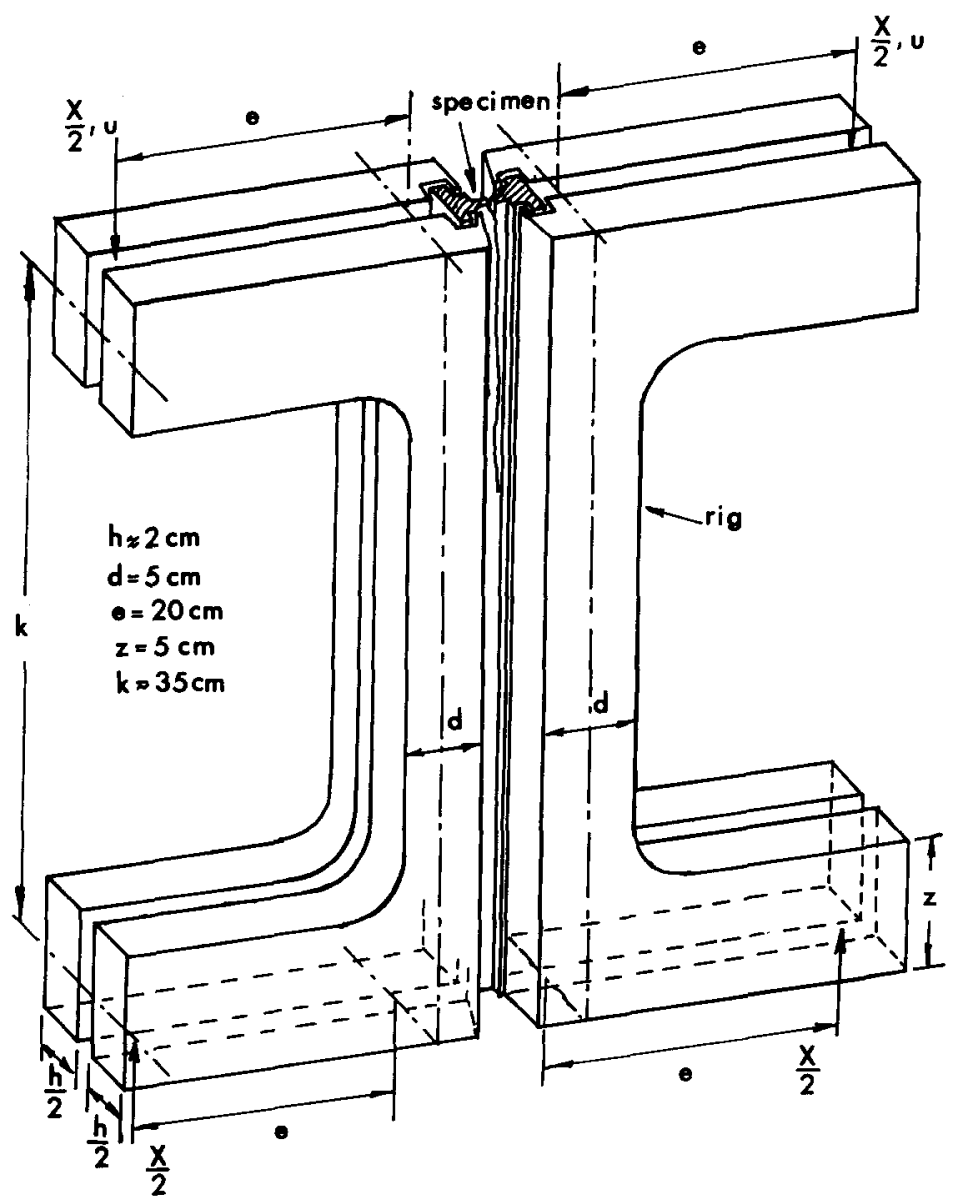

Figure 3a. A simple crack propagation apparatus for fracture toughness determination (Gurney, Mai and Owen [1]). 
respectively in [1] and [17]. Briefly, it consists of two beams of a high yield strength material coupled with the testpiece which acts as the web of a flanged beam. Fracture toughness or $R$-curve determination may be obtained by causing a crack to extend down the centre of the testpiece. A dimensioned cross-section of the $I$-beam testpiece is also shown in Fig. 3b. Note that the specimen is prepared initially from a rectangular bar to the $I$-geometry and the test section machined to the required thickness $(B)$. Thus, in principle, $R$-curves for materials with various thicknesses can be obtained. However, as mentioned earlier in the introduction, due to the limitations of the machine capacity, only thin sheets (i.e. $0.76 \mathrm{~mm}$ thick) have been studied in the present investigation.
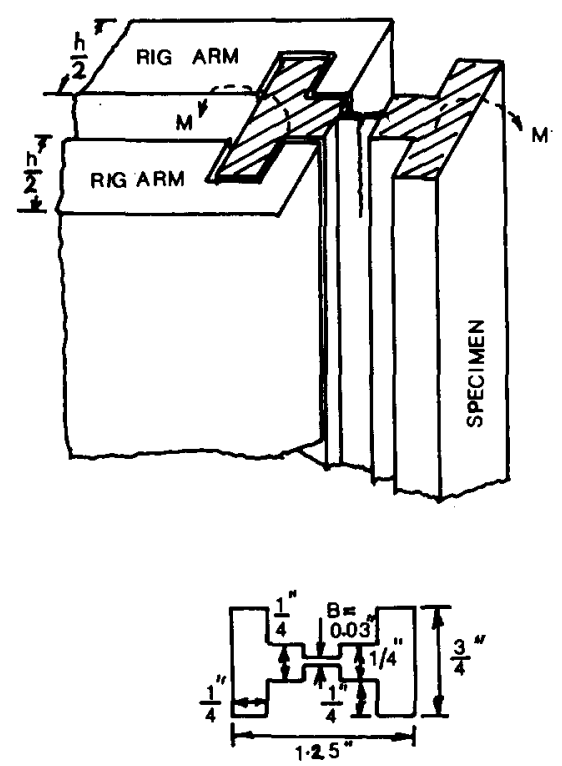

Figure 3b. Dimensioned cross-section of a typical testpiece (after Owen et al. [17]).

The following analysis of cracking in terms of "beams on elastic foundations" is similar to that of Kanninen [18], who has used the model to study dynamic fracture. Gurney, Mai and Owen [1] and Mai [19] have independently used an "elastic foundation" model of cracking to investigate the stability of crack propagation $[1,7,19,20]$. Fracture in the present case may be modelled by the breakage of linear elastic springs under the action of two equal and opposite bending moments $(M)$ applied at its ends. With reference to Fig. 4 and assuming quasi-static extension of the crack takes place at constant end slope of the beam $(\theta)$, we have from (3):

$$
B R=-(\partial \Lambda / \partial L)_{0}
$$

where $\Lambda$ is expressed as:

$$
\Lambda=\int_{0}^{W} E I\left(\frac{\mathrm{d}^{2} v}{\mathrm{~d} x^{2}}\right)^{2} \mathrm{~d} x+\int_{0}^{W} k v^{2} \mathrm{~d} x
$$

Thus:

$$
B R=-\frac{\partial}{\partial L} \int_{0}^{W} E I\left(\frac{\mathrm{d}^{2} v}{\mathrm{~d} x^{2}}\right)^{2} \mathrm{~d} x-\frac{\partial}{\partial L} \int_{0}^{W} k v^{2} \mathrm{~d} x
$$

where $B$ is the effective thickness of the fracture plane and $k$ the stiffness of the half springs per unit length of the beam on the foundation. By interchanging differentiation 


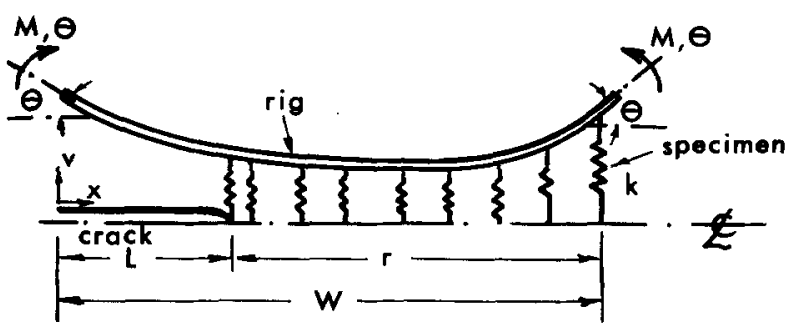

Before crocking

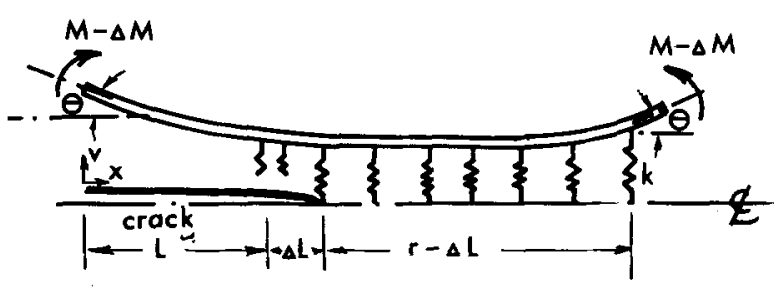

After cracking

Figure 4. Cracking model for rig and testpiece.

and integration for this expression, and performing integration by parts with boundary conditions (see Fig. 4):

$$
\begin{aligned}
& v^{\prime}(0)=-v^{\prime}(W)=\theta \\
& v^{\prime \prime}(0)=-v^{\prime \prime}(W)=M /(E I) \\
& v^{\prime \prime \prime}(0)=-v^{\prime \prime \prime}(W)=0
\end{aligned}
$$

this gives:

$$
B R=-2 \int_{0}^{W} \frac{\partial v}{\partial L}\left[E I \frac{\mathrm{d}^{4} v}{\mathrm{~d} x^{4}}+k v\right] \mathrm{d} x+k v_{x=L}^{2}
$$

where $v_{x=L}$ is the relative opening displacement of the beam at crack tip section. However, from beam on elastic foundation theory:

$$
E I\left(\mathrm{~d}^{4} v / \mathrm{d} x^{4}\right)+k v=0 .
$$

Therefore, for quasi-static crack extension to occur, the relative opening displacement of the beam $\left(v_{x=L}\right)$ must reach a critical value $v_{c}$ so that:

$$
k v_{\mathrm{c}}^{2}=R B \text {. }
$$

The relative opening displacement of the beam at the crack tip section may be found in Hetenyi [21] as:

$$
v=\frac{2 M \lambda^{2}}{k} \frac{\sinh (\lambda r)-\sin (\lambda r)}{\sinh (\lambda r)+\sin (\lambda r)}
$$

where $r$ is the length of the unbroken ligament of the beam testpiece. $\lambda$, the reciprocal of characteristic length of beam on the foundation, is defined by:

$$
\lambda^{4}=k / 4 E I
$$


Hence, combining (13), (14) and (15), the bending moment $\left(M_{c}\right)$ to cause quasi-static cracking will be given by:

$$
\frac{M_{c}}{(R E I B)^{\frac{1}{2}}}=\frac{\sinh (\lambda r)+\sin (\lambda r)}{\sinh (\lambda r)-\sin (\lambda r)}
$$

Figure 5 shows the relation of $M_{c} /(R E I B)^{\frac{1}{2}}$ to $(\lambda r)$.

To determine the $R$-curve, it is necessary to use either (13) or (16) to record continuously the beam opening displacement $\left(v_{c}\right)$ or the critical bending moment $\left(M_{c}\right)$ as a function of $(\lambda r)$.

Through the reinforcement of the testpiece, by the high yield strength test rig, general yielding at regions other than the crack tip is avoided. This enables the valid crack growth resistance $(R)$ of a cracked structure to be determined.

Cracking before general yielding of the testpiece in such an apparatus (see Fig. 3) occurs when [1]:

$$
(h d)_{\mathrm{rig}}>\left(3 E / \sigma_{y}^{2}\right)_{\mathrm{rig}} \cdot(R B)_{\text {testpiece }}
$$

Consider a high tensile steel rig with $E=210 \mathrm{GN} / \mathrm{m}^{2}$ and $\sigma_{y}=1.66 \mathrm{GN} / \mathrm{m}^{2}$ which is designed to test a "tough" 2024 aluminum plate of thickness $(B) 0.05 \mathrm{~m}$, where $R=196$ $\mathrm{kJ} / \mathrm{m}^{2}$ and $\sigma_{y}=98 \mathrm{MN} / \mathrm{m}^{2}$. The minimum cross-sectional area of the rig $(h d)$ calculated from (17) is $0.002 \mathrm{~m}^{2}$. However, if there is no reinforcement, as provided by the rig,

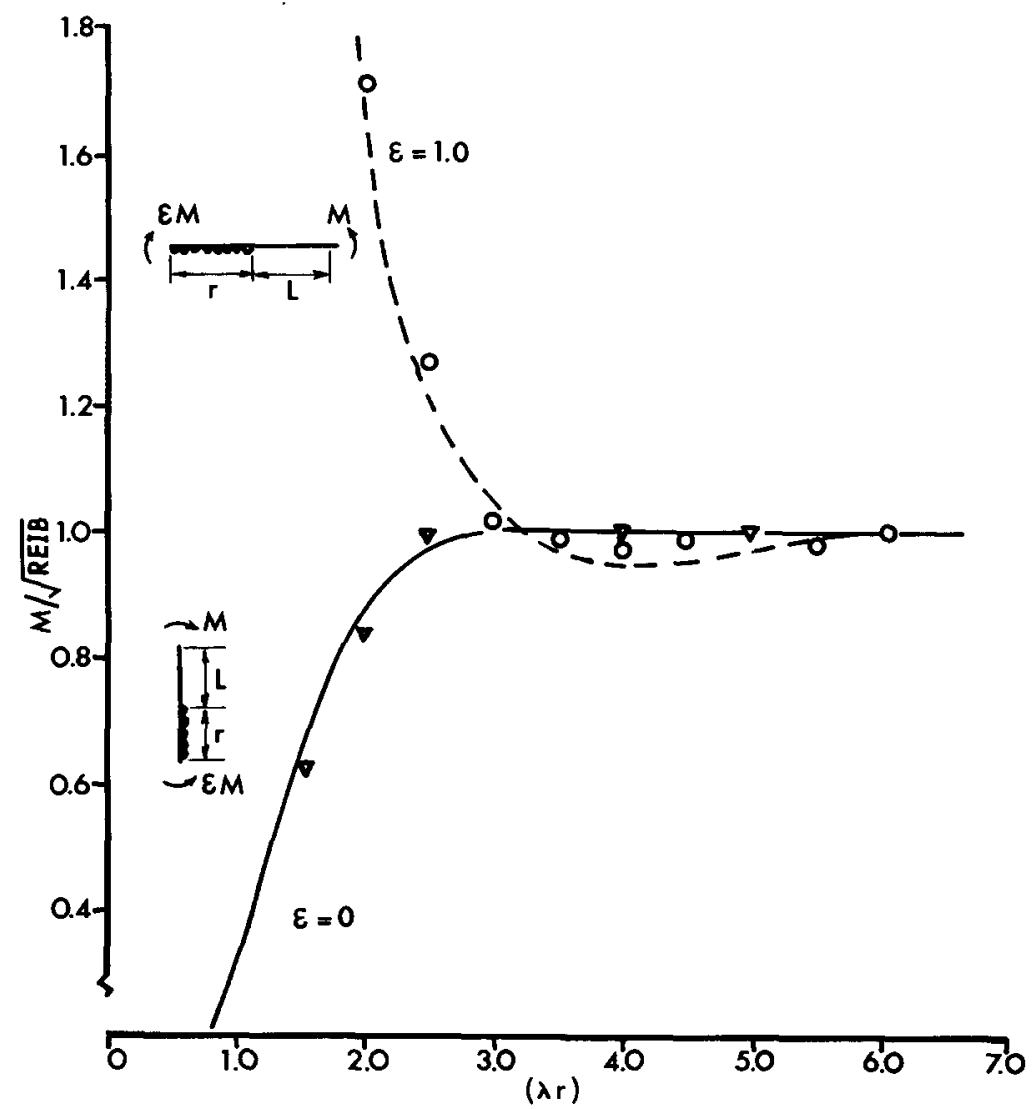

Figure 5. Relationship between critical bending moment $\left(M_{c}\right)$ to cause quasi-static crack extension, and $\lambda r$, the crack length. 
and if a conventional beam-like testpiece is employed, the necessary half beam depth obtained from (1) will be $4.50 \mathrm{~m}$. This gives a minimum testpiece cross-sectional area of $2 B d=0.450 \mathrm{~m}^{2}$. The practical impossibility of such a laboratory test size is obvious.

Proper design of the rig should allow ready splitting of plate specimens for a range of thickness $(B)$ from fractions of a millimetre to approximately $50 \mathrm{~mm}$ in a 100 ton testing machine. This provides a definite means for probing into the effect of thickness or fracture mode transitions on the $R$-curve.

The test configuration also provides better stability in cracking a material whose behaviour is characterised by negative $\mathrm{d} R / \mathrm{d} L$ or $\mathrm{d} R / \mathrm{d} L[1,19,20]$ and hence entails the complete determination of the $R$-curve. It may be shown that in displacementcontrolled machines, the geometrical stability factor is large as crack extension proceeds $[1,19]$.

\section{Experimental results and discussion}

In the experiments, the testpieces were fitted to the slots of the rig and loaded as shown in Fig. 3. The critical load, or bending moment $\left(M_{c}\right)$ to cause quasi-static cracking was recorded as the crack extension was monitored by a travelling microscope. Critical opening displacement of the beam $\left(v_{c}\right)$ may also be recorded by mounting two clip-on guages, one in front and the other at the back of the rig directly over the crack tip.

Figure 6 shows the $R$-curve of a $0.76 \mathrm{~mm}$ thick 7075 aluminium alloy obtained by the use of this cracking apparatus. The Young's modulus and the yield stress for this metal are $73.5 \mathrm{GN} / \mathrm{m}^{2}$ and $196 \mathrm{MN} / \mathrm{m}^{2}$, respectively. Two complete $R$-curves with different starter-crack lengths of $0.5 \mathrm{~cm}$ and $11.0 \mathrm{~cm}$, respectively, are presented. It

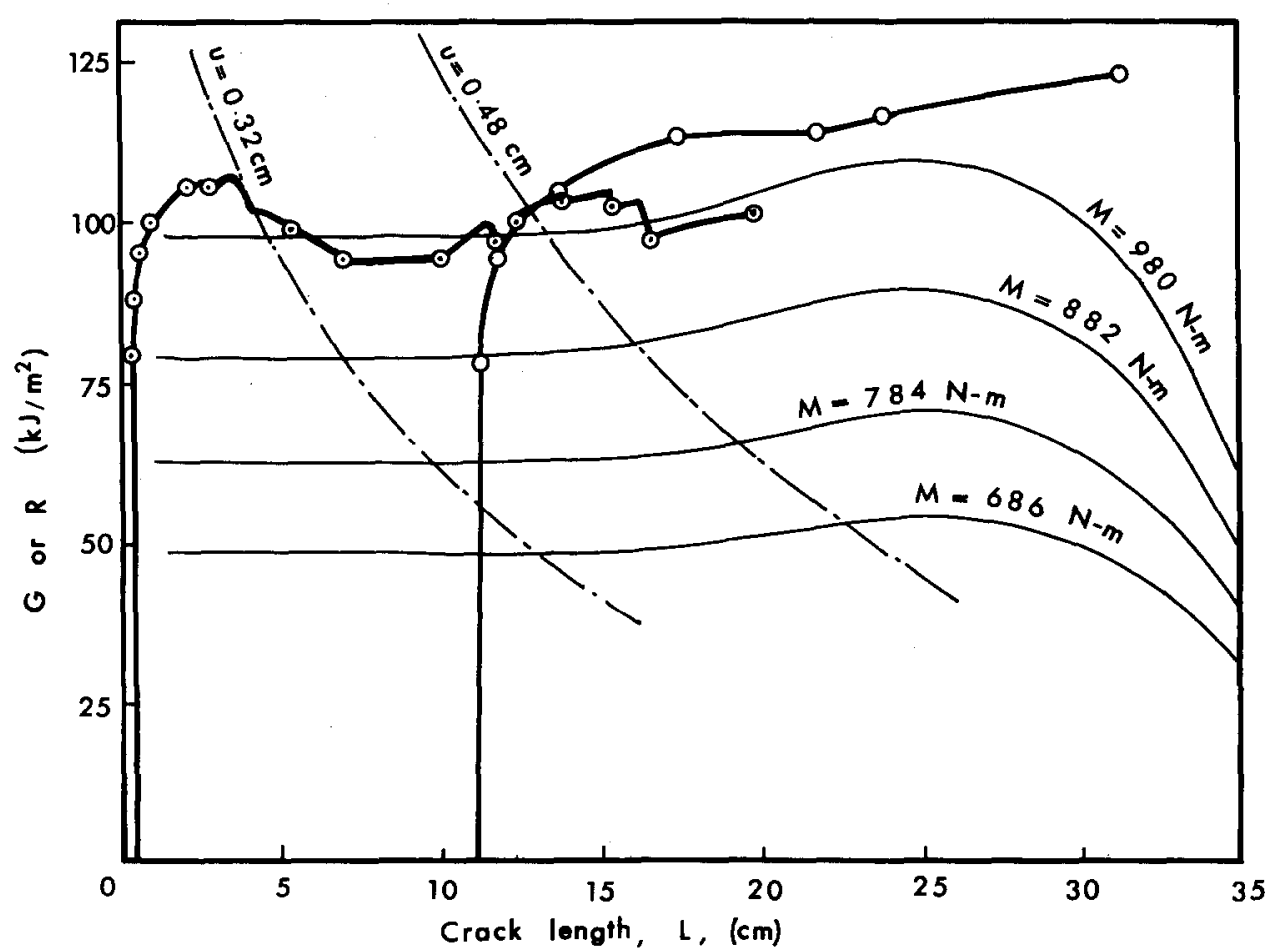

Figure 6. Crack growth resistance $(R)$ and strain energy release rate $(G)$ curves for a 7075 aluminium alloy, $0.762 \mathrm{~mm}$ thick. 


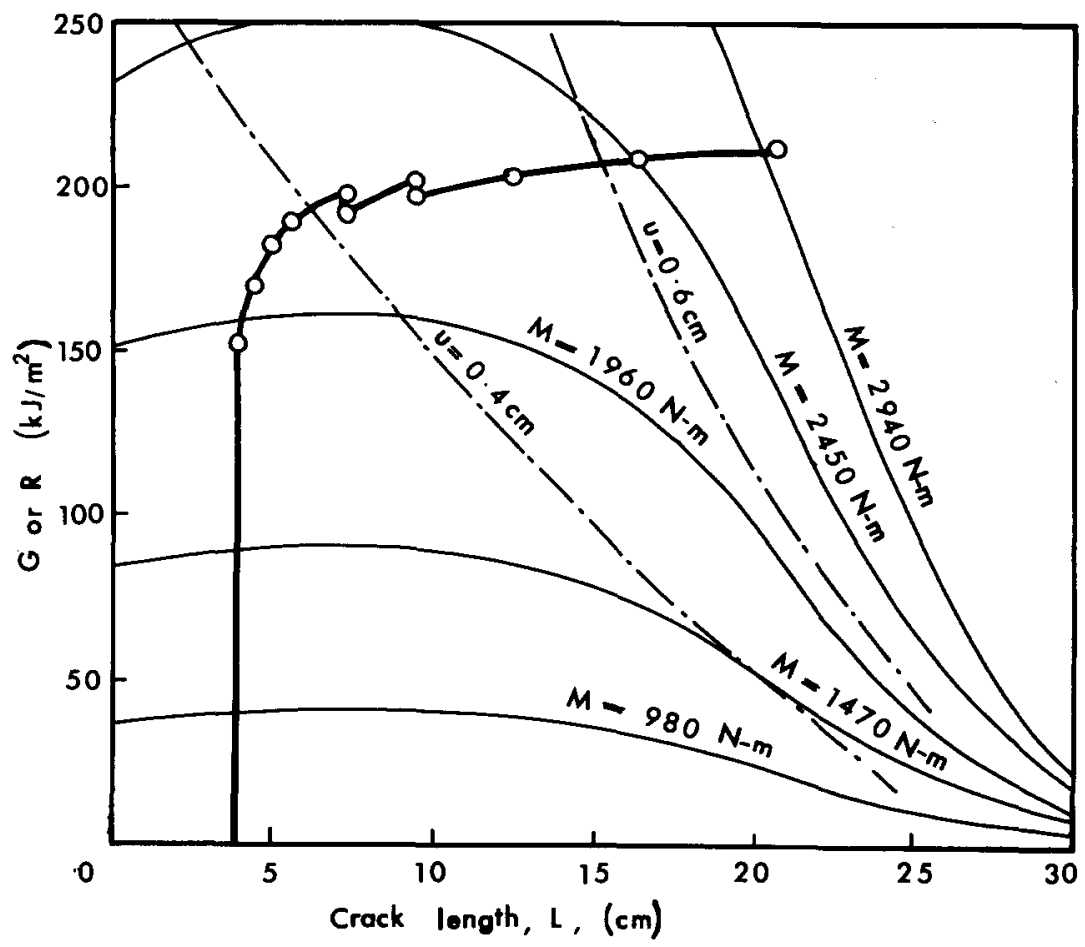

Figure 7. Crack growth resistance $(R)$ and strain energy release rate $(G)$ curves for a low carbon steel, $0.762 \mathrm{~mm}$ thick.

may be seen that in the slow stable growth region, the crack growth resistance rises gradually with crack extension and is independent of the initial crack length. The variation of fracture toughness in the subsequent crack growth is probably associated with the effects of crack front velocities and local conditions at the crack tip. Instabilities of crack spreading are marked by the drop of $R$ at constant crack length $(L)$. A maximum toughness of $105 \mathrm{~kJ} / \mathrm{m}^{2}$ for the material has been obtained, which, since the fracture plane shows necking to a sharp line, is deemed to represent a plane stress value. The features of the $R$-curve for a thin low carbon steel with $E=210 \mathrm{GN} / \mathrm{m}^{2}$ and $\sigma_{y}=274 \mathrm{MN} / \mathrm{m}^{2}$ is shown in Fig. 7. The negative $\mathrm{d} R / \mathrm{d} \dot{L}$ behaviour of mild steel in the continuous growth region at room temperature is indicated by the instabilities on the $R$-curve at the early part of crack extension. It may be seen that crack stability is greatly improved as the crack extends (i.e. where $\lambda r<\pi$ [1]). The fracture plane also shows necking with a maximum plane stress fracture toughness of about $215 \mathrm{~kJ} / \mathrm{m}^{2}$. Figure 8 gives the complete $R$-curve for a commercial 1100 aluminium alloy of 0.762 $\mathrm{mm}$ net section thickness. Note that there is a region of enormous stable crack extension before the plateau is reached. This gives a values of $168 \mathrm{~kJ} / \mathrm{m}^{2}$ for the commercial aluminium.

The improvement of stability provided by this apparatus over conventional test methods $[1,8,19]$ in either a soft or a hard testing machine may be visualised from the $R$-curve and the $(G, L)_{M}$ or $(G, L)_{\mu}$ curves. Therefore, it should enable the determination of complete $R$-curves for most unstable materials characterised by negative $\mathrm{d} R / \mathrm{d} \dot{A}$.

Finally, a discussion on the possible effects on these $R$-curves due to the interaction of the crack tip plastic zone and the flanges of the specimen is necessary. If the plastic zone size $(\rho)$ is allowed to fully develop to its maximum value, $\rho$ is approximately 0.25 $\left(K_{\mathrm{c}} / \sigma_{y}\right)^{2}$ and is of the order of inches for these high $\left(K_{\mathrm{c}} / \sigma_{y}\right)^{2}$ materials. Since the face groove widths of all our specimens are roughly $6 \simeq 7 \mathrm{~mm}$ and are much less than $\rho$, 


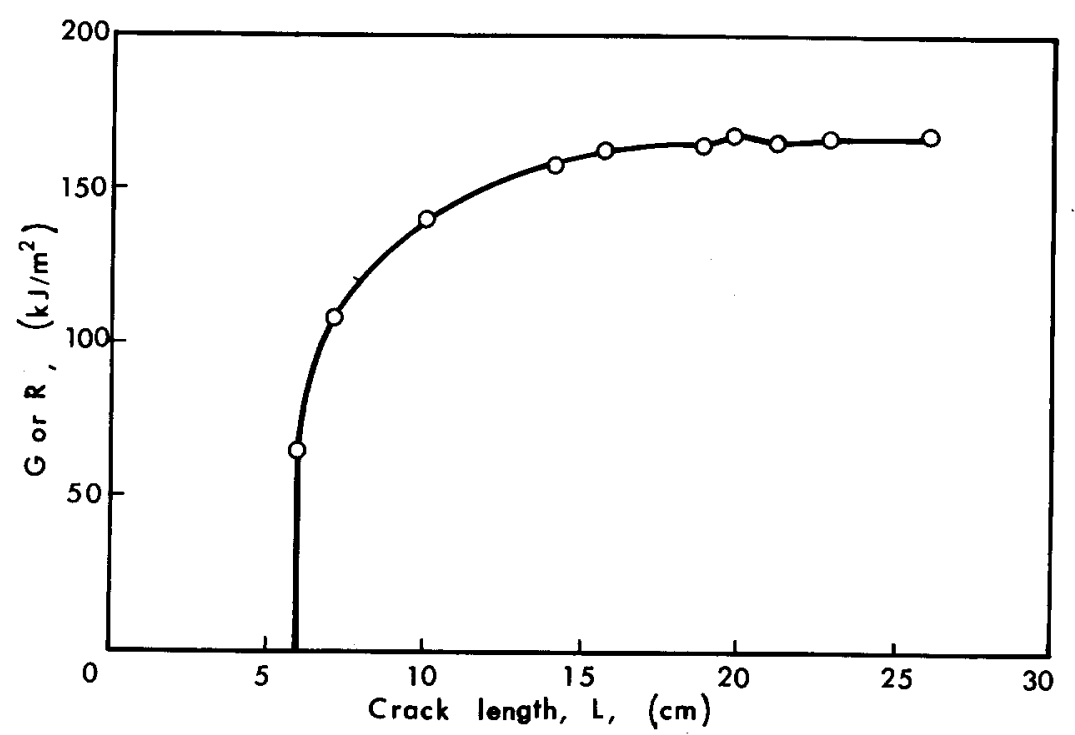

Figure 8. Crack growth resistance $(R)$ curve for $1100-0$ aluminium alloy, $0.762 \mathrm{~mm}$ thick.

some interactions between the specimen flanges and the plastic zone must have occurred. Admittedly, this implies that the $R$-curves have also been affected by this effect. However, it is thought that, due to the constraints provided by the much thicker flanges of the test rig compared with the test section thickness (this ratio $(h / B)$ being $\simeq 500), \rho$ is reduced considerably because the plastic zone now cannot grow freely to its otherwise full size as when there are no constraints. (This, in fact, is the original intention of the design of the test rig [1] which is expected to simulate quasi-brittle fracture behaviour in these otherwise ductile materials.) Naturally, depending on the width of the web section of the testpiece, and also the ratio of the test section thickness to that of the reinforcements (i.e. the flanges), which are significant parameters in controlling the amount of interaction, the $R$-curves can be obtained between the limits of plane strain/stress situations. In the present investigation, these parametric effects on $R$-curves of high $\left(K_{\mathrm{c}} / \sigma_{y}\right)^{2}$ materials have not been systematically examined. It is expected that this will be the subject of a future investigation which should shed more light on the $R$-curve research technologies. At this point, it should be mentioned that in our experiments the plastic zone geometry observed at the crack tip resembles that of a Dugdale yield strip $^{\star}$. Also, irreversible stretched deformations with necking to a sharp line at the fractured surfaces have been noted in the thin grooved test section. We speculate, therefore, that the $R$-curves we have obtained for the aluminium alloys and the low carbon steel must be close to the plane stress deformation situation.

\section{R-curve geometry}

As postulated by many workers, the crack growth resistance during slow stable subcritical crack extension (i.e. region 1 in Fig. 1) seems to be merely a function of $\Delta L$. Empirical relations such as a simple power law, $R=F(\Delta L)^{p}$, and a second order polynomial of the form, $R=C_{0}+C_{1} \Delta L+C_{2}(\Delta L)^{2}$, to relate $R$ and $\Delta L$ have been suggested by Broek [14-16] and Wang and McCabe [2], respectively. While the Broek relation is simple, it does say that at $\Delta L \simeq 0, R \simeq 0$ which means that cracks start to propagate as soon

\footnotetext{
$\star$ In general, the geometry of the plastic zone should depend on the web section width and thickness as well as the work hardening index of the material under consideration. It is expected that plastic zone geometry in the form of a Dugdale yield strip should minimise the interaction effect discussed above.
} 


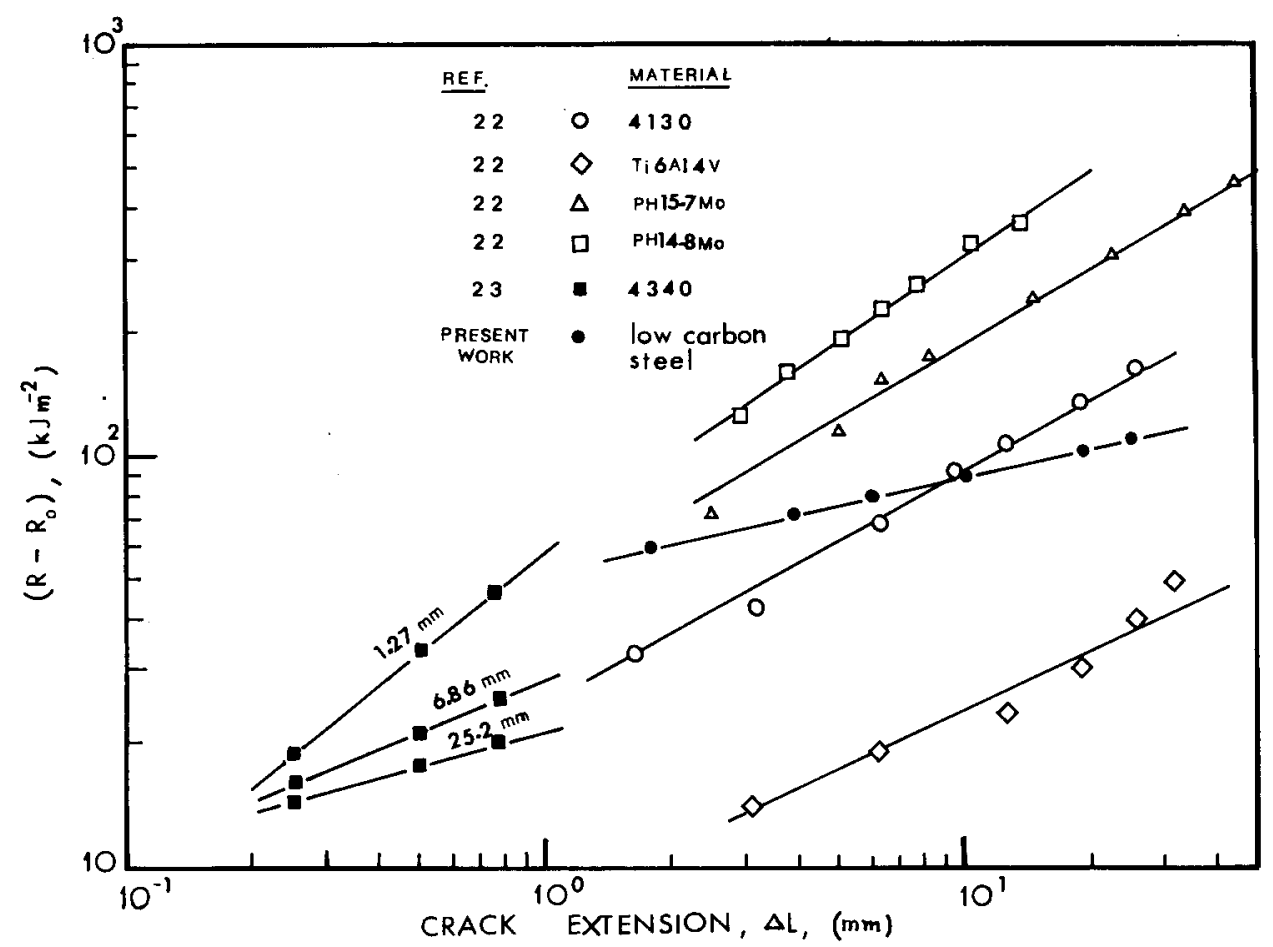

Figure 9. Crack growth resistance curves for steels.

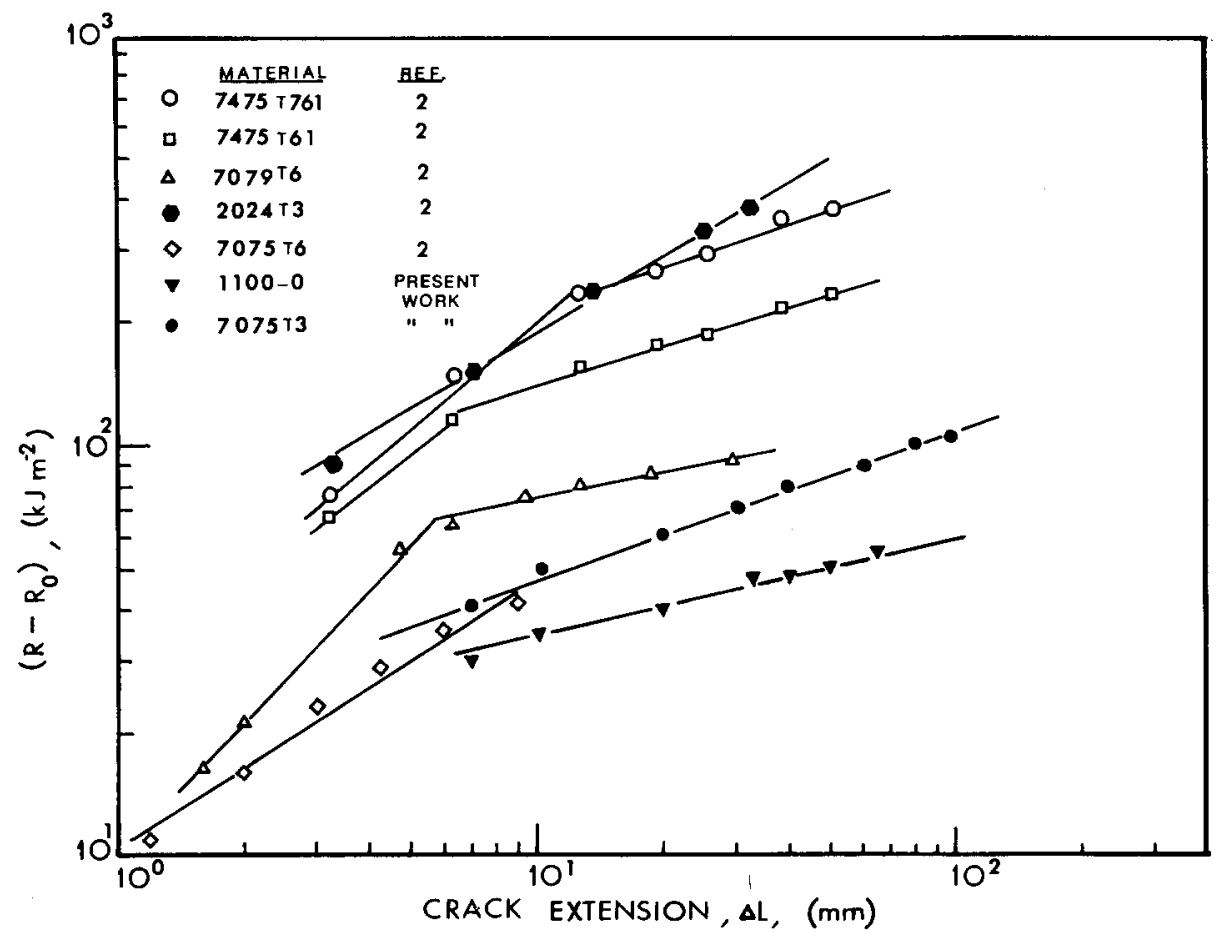

Figure 10. Crack growth resistance curves for aluminium alloys. 


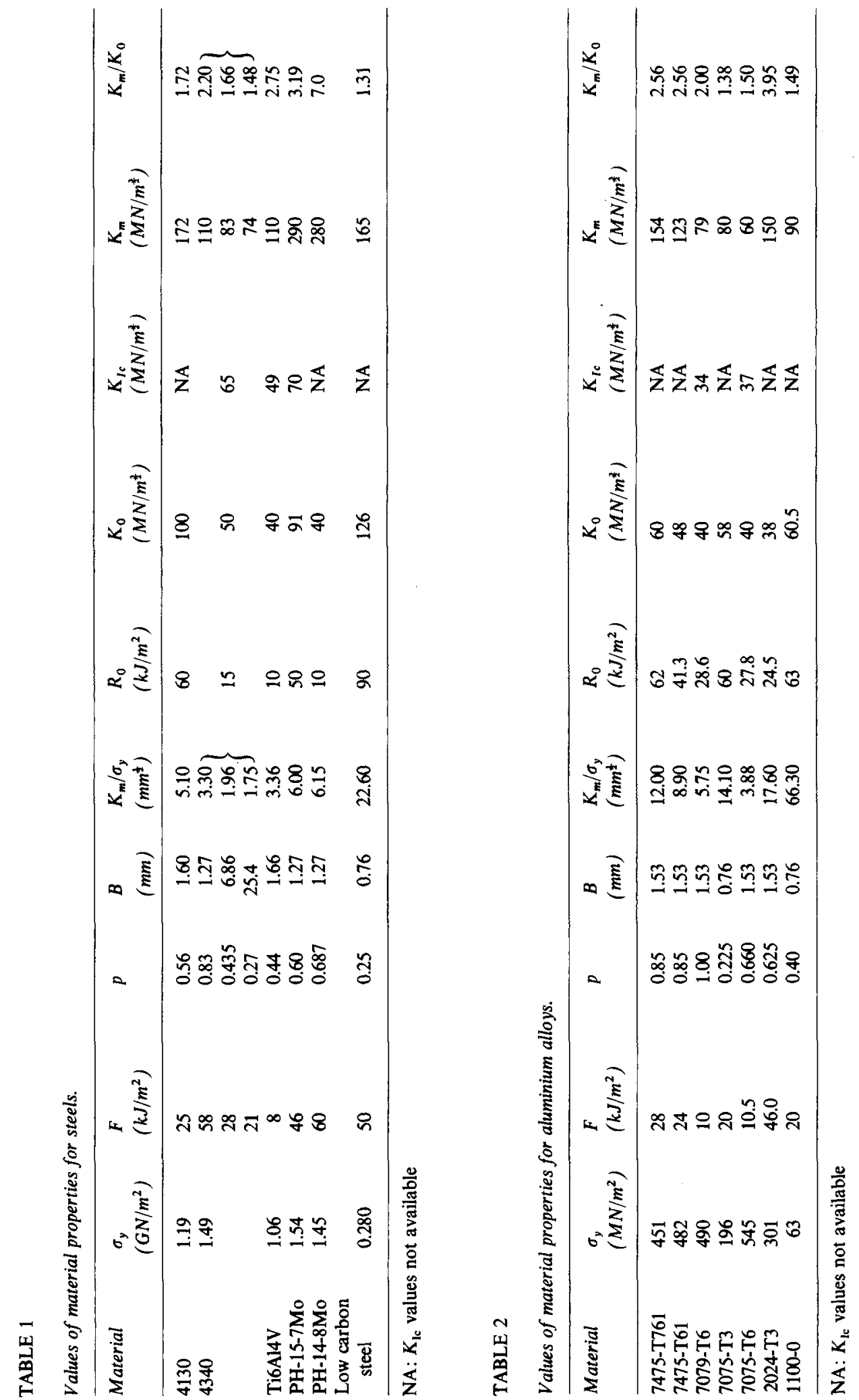


as the specimen is loaded. This boundary condition can hardly be general because inspection of many published $R$-curves for various heat-treated steels and aluminium alloys (e.g. ASTM STP No. 527) show that at $\Delta L \simeq 0$, a finite value of $R=R_{0}$ exists, which is caused by the work of generating the plastic zone before appreciable crack extension.

A simple correlation between $R$ and $\Delta L$ may be:

$$
R=R_{0}+F(\Delta L)^{p}
$$

which has been tested using previously published $R$-curve data $[2,22]$ and the present experimental results. Figures 9 and 10 show plots (of $\log \left(R-R_{0}\right)$ versus $\log (\Delta L)$ ) for various steels and aluminium alloys where $R_{0}$ values $\left(=K_{0}^{2} / E\right)$ have been obtained by back-extrapolation to the $R$-axis or $K$-axis when $(\Delta L) \simeq 0$. Despite some admitted uncertainty in this latter procedure, it may be seen that (18) is, in general, a reasonably good representation for the relation between $R$ and $\Delta L$. However, it should be noted that the aluminium alloys 7079-T6, 7475-T61 and 7475-T761 all display slope transitions in these plots, the slopes diminishing at large $\Delta L$, which means that the relationship is not as simple as we may wish. Similar changes in slope are observed if simple $\log R$ is plotted against $\log \Delta L$ (i.e. Broek's equation) for these same materials.

Tables 1 and 2 summarise the values of $F, p, R_{0}$, etc., for these steels and aluminium alloys. $K_{m}$ is the value of the stress intensity factor on the plateau of the $R$ versus $\Delta L$ plot.

$$
R-R_{0}=F(\Delta L)^{p}
$$

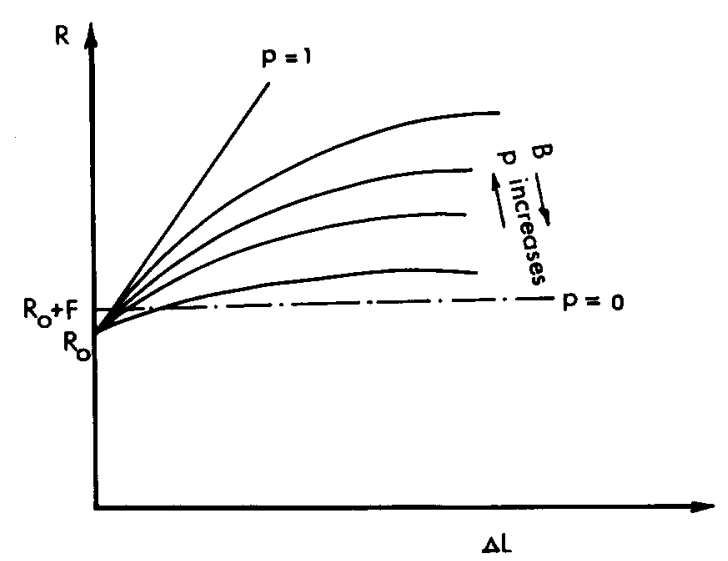

Figure 11. A sketch of $R$-curves as a function of $p$.

Figure 11 is a schematic representation of the $R$-curve as a function of $p$. When $p=0$, the $R$-curve is a step function of value $\left(R_{0}+F\right)$. The geometry of the $R$-curve must be a function of the stress state at the crack tip, i.e. how far away from or close to plane strain conditions is the test. In this way, we would expect $K_{m}$ to coincide with $K_{\mathrm{Ic}}$ when $B$ was thick enough to satisfy the plane strain thickness requirement, i.e. $B>2.5\left(K_{\mathrm{Ic}} / \sigma_{y}\right)^{2}$. In general terms, therefore, $\left(K_{m} / K_{\mathrm{Ic}}\right)$ should decrease to unity as $B \div 2.5\left(K_{\mathrm{Ic}} / \sigma_{y}\right)^{2}$ increases. We may also argue that the $R$-curve for plane strain is essentially a step function, with $R$ independent of $\Delta L^{\star}$. This occurs as $p \rightarrow 0$, when $R \rightarrow R_{0}+F$. We might therefore expect an inverse relationship between $p$ and $\{B \div 2.5$

« This excludes those apparently plane strain cases where $R$ increases during propagation, even though $B>2.5$ $\left(K_{\mathrm{tc}} / \sigma_{y}\right)^{2}[23]$. It is arguable that this could be caused by changes in $R$ with crack speed, i.e. $\mathrm{d} R / \mathrm{d} \dot{A}$ positive. 
$\left.\left(K_{\mathrm{Ic}} / \sigma_{y}\right)^{2}\right\}$ or a direct relationship between $p$ and $\left(K_{m} / K_{\mathrm{Ic}}\right)$. Again, it seems that $F$ gets smaller as $B$ increases or $\left(K_{\mathrm{Ic}} / \sigma_{y}\right)^{2}$ decreases. This suggests perhaps surprisingly that $R_{0}$ may correspond with $R$ for plane strain fracture (i.e. $G_{\mathrm{Ic}}$ ). It certainly seems odd at first sight that plane stress fractures should initiate at the same $R$ level as plane strain (given the wide differences in stress state and fully developed plastic zone size). Yet, the fully developed zone does not occur until $K_{m}$ is reached, so perhaps there is always enough constraint in the middle of a thin sheet to initiate cracks at $K_{0}$. Also, experimentally, all $R$-curves do tend to emanate from a common $R_{0}$. The subsequent development of a larger plastic zone than the plane strain zone comes about under rising $R$ since subsequent lack of constraint prevents much propagation at lower $R$ values. This point is not altogether clear to us. Nevertheless, Tables 1 and 2 show that values of $K_{0}\left(=\left(E R_{0}\right)^{\frac{1}{2}}\right)$ and $K_{\mathrm{Ic}}$ (where known) are in good agreement. Many $K_{\mathrm{Ic}}$ values are not available because of experimental difficulties in meeting size requirements, as is well known, and of course the $R$-curve technique has often been employed in thin sheets of tough materials. The $K_{0}$ and $R_{0}$ values for materials where $K_{\mathrm{Ic}}$ is not available are reasonable.

Approximating $K_{\mathrm{Ic}}$ by $K_{0}$, we can test the foregoing suggested relations for the dependence of $K_{m}, p$ and $F$ on $B, K_{0}$ and $\sigma_{y}$. There is undoubted scatter in such plots, and attempts to bring $p, F, K_{m}, B, K_{\mathrm{lc}}$ and $\sigma_{y}$ all together have not been successful. Nevertheless, in broadest terms:

$$
p=\ln \left(K_{m} / K_{0}\right)
$$

$F$ and $\left(K_{m} / K_{0}\right)$ generally go down as $B \div 2.5\left(K_{0} / \sigma_{y}\right)^{2}$ increases. Clearly, the correct relationships involve in addition such quantities as work hardening indices, most of which are unknown.

However, if the concept that $K_{0} \simeq K_{\mathrm{Ic}}$ is reasonable, it seems to open up a possible method of determining $K_{\mathrm{Ic}}$ from $R_{0}$ values, at least to a good approximation.

\section{Conclusions}

In the present paper, a novel approach for measuring $R$-curves has been presented. The small size laboratory apparatus has proved successful for the determination of valid $R$-curves for high toughness and low yield strength materials $\left(\left(K_{m} / \sigma_{y}\right)^{2}\right.$ for the 7075 - and 1100-aluminium alloys and steels are $200 \mathrm{~mm}, 4.4 \mathrm{~m}$ and $500 \mathrm{~mm}$, respectively). Several other advantages, inherent in this cracking apparatus, should be mentioned.

(a) It allows the collection of complete $R$-curves for ductile metals as a function of a range of material thickness. Fracture transition associated with thickness effects may therefore be investigated.

(b) Better stability is provided for cracking materials which are characterised by negative $\mathrm{d} R / \mathrm{d} L$. Therefore, $R$-curves for rate sensitive materials will be easily constructed.

(c) Testpiece size requirements for valid tests on ductile materials are reduced with the use of this design.

(d) The cracking system is essentially displacement reversible. It permits easy monitoring of critical cracking load and opening displacement of the beam at the crack tip as the crack extends.

Both the $R$-curves obtained from this apparatus and those determined by other means seem to fit the simple relation:

$$
R=R_{0}+F(\Delta L)^{p}
$$

where $F$ and $p$ are functions of material, sheet thickness and $\left(K_{0} / \sigma_{y}\right)^{2}$ ratio. $K_{0}\left(=\left(E R_{0}\right)^{\frac{1}{2}}\right)$ 
values seem to agree quite well with $K_{\text {Ic }}$ values, where known, so that an approximate method of determining $K_{\mathrm{Ic}}$ values may be available, especially for materials possessing extremely large $\left(K_{\mathrm{Ic}} / \sigma_{y}\right)^{2}$ ratios.

\section{Acknowledgement}

The authors would like to express their thanks to Professor C. Gurney, Head of the Department of Mechanical Engineering, University of Hong Kong, for permission for one of them (YWM) to use their equipment for the $R$-curve experiments and for stimulating discussions.

\section{REFERENCES}

[1] C. Gurney, Y. W. Mai and R. C. Owen, Proceedings of the Royal Society (London) A340 (1974) $213-231$.

[2] D. Y. Wang and D. E. McCabe, Investigation of R-curve using comparative tests with CCT and CLWL specimens, presented at Eight National Fracture Symposium, Brown University, August 1974.

[3] R. H. Heyer and D. E. McCabe, Engineering Fracture Mechanics, 4 (1972) 413-430.

[4] R. W. Judy, Jr. and R. J. Goode, in Fracture Toughness Evaluation by R-curve Methods, ASTM STP No. 527 (1973) 58-61.

[5] C. Gurney and J. Hunt, Proceedings of the Royal Society (London), A299 (1967) 508-524.

[6] C. Gurney and K. M. Ngan, ibid., A325 (1971) 207-222.

[7] C. Gurney and Y. W. Mai, Engineering Fracture Mechanics, 4 (1972) 853-863.

[8] D. P. Clausing, International Journal of Fracture Mechanics, 5 (1969) 211-227.

[9] E. J. Ripling and E. Falkenstein, in Fracture Toughness Evaluation by R-curve Methods, ASTM STP No. 527 (1973) 36-47.

[10] G. T. Hahn, M. Sarrate and A. R. Rosenfield, International Journal of Fracture Mechanics, 7 (1971) $435-446$.

[11] Proposed Recommended Standard for $R$-Curve Determination, Committee E-24 ASTM, E24.01.04, Task Group on $R$-Curves.

[12] C. Gurney, in Physical Basis of Yield and Fracture, ed. A. C. Strickland, Institute of Physics and Physical Society, London (1967) 8.

[13]J. E. Srawley and W. F. Brown, Jr. in Fracture Toughness Testing and Its Applications, ASTM STP No. 381 (1965) 133-198.

[14] D. Broek, Engineering Fracture Mechanics, 5 (1973) 45-53.

[15] D. Broek, The Residual Strength of Aluminium Sheet Alloy Specimens Containing Fatigue Cracks or Saw Cuts, Tech. Rept. NRL-TR M. 2143, National Aerospace Laboratory, Amsterdam (1965).

[16] D. Broek, The Effect of Finite Specimen Width on the Residual Strength of Light Alloy Sheet, Tech. Rept. NRL-TR M. 2152, National Aerospace Laboratory, Amsterdam (1965).

[17] R. C. Owen, Y. W. Mai and C. L. Chow, International Journal of Fracture, 12 (1976) 3-17.

[18] M. F. Kanninen, International Journal of Fracture, 9 (1973) 83-92.

[19] Y. W. Mai, International Journal of Fracture, 12 (1976) 107-123.

[20] Y. W. Mai, International Journal of Fracture, 9 (1973) 349-351.

[21] M. Hetenyi, Beams on Elastic Foundation, University of Michigan Press, Ann Arbor (1947).

[22] A. M. Sullivan, C. N. Freed and J. Stoop in Fracture Toughness Evaluation by R-Curve Method, ASTM STP No. 527 (1973) 85-104.

[23] M. H. Jones and W. F. Brown in Review of Developments in Plane Strain Fracture Toughness Testing, ASTM STP No. 463 (1970) 63-91. 